\title{
Jacques Cherblanc (éd.), Rites et symboles contemporains, théories et pratiques
}

Québec, Presses de l'Université du Québec, 2011, 206 p.

\section{Mira Niculescu}

\section{(2) OpenEdition}

\section{Journals}

Édition électronique

URL : http://journals.openedition.org/assr/24259

DOI : $10.4000 /$ assr. 24259

ISSN : $1777-5825$

Éditeur

Éditions de l'EHESS

Édition imprimée

Date de publication : 30 décembre 2012

Pagination : 142

ISSN : 0335-5985

Référence électronique

Mira Niculescu, « Jacques Cherblanc (éd.), Rites et symboles contemporains, théories et pratiques », Archives de sciences sociales des religions [En ligne], 160 | octobre-décembre 2012, mis en ligne le 21

février 2013, consulté le 21 septembre 2020. URL : http://journals.openedition.org/assr/24259; DOI https://doi.org/10.4000/assr.24259

Ce document a été généré automatiquement le 21 septembre 2020.

(c) Archives de sciences sociales des religions 


\section{Jacques Cherblanc (éd.), Rites et symboles contemporains, théories et pratiques}

Québec, Presses de l'Université du Québec, 2011, 206 p.

Mira Niculescu

\section{RÉFÉRENCE}

Jacques Cherblanc (éd.), Rites et symboles contemporains, théories et pratiques, Québec, Presses de l'Université du Québec, 2011, 206 p. 
1 «Rites et symboles contemporains » est un ouvrage collectif publié en 2011 à l'initiative $\mathrm{du}$ Lerars, le Laboratoire d'Expertise et de Recherche en Anthropologie Rituelle et Symbolique auprès de l'Université du Québec. Il entend offrir au lecteur, universitaire, professionnel et grand public, un panorama des "théories et pratiques" rituelles contemporaines.

2 À travers une « réflexion sur la place et le rôle des rites et des symboles dans nos sociétés contemporaines » (Cherblanc, p. 2) les différentes études de cas présentées visent à identifier pragmatiquement «qui » manipule « quels » symboles, et à partir de « quelles réserves » symboliques. En effet, l'objectif affiché du Lerars est de nourrir les savoir-

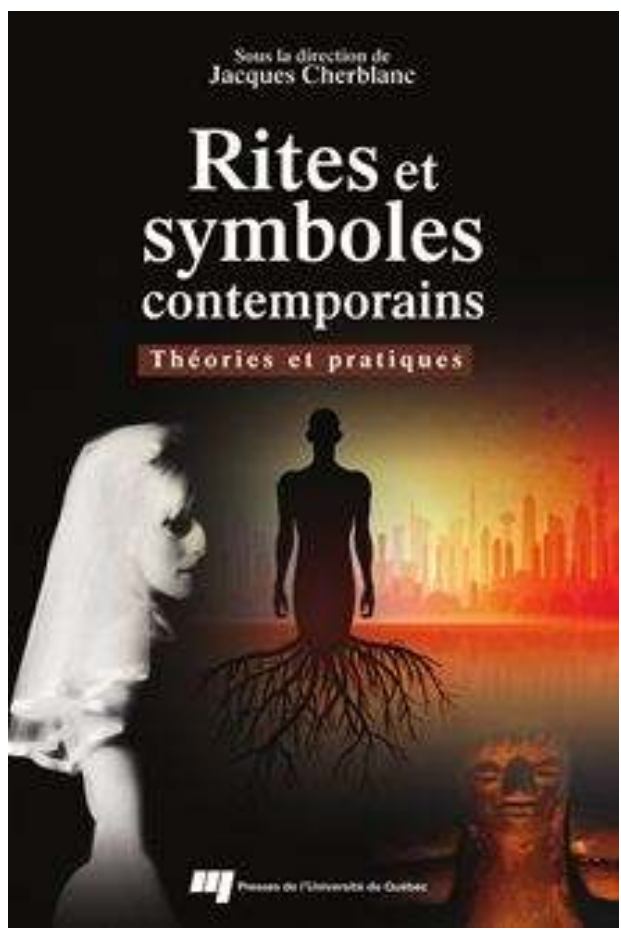
faire de terrain tout autant que la réflexion théorique : loin de se contenter d'analyser la "mise en sens aujourd'hui ", comme titrait la conférence préalable à cet ouvrage, le laboratoire se donne pour vocation prescriptive de mettre au point de nouvelles pratiques rituelles à proposer aux intervenants sociaux, hospitaliers, éducatifs...

3 La collaboration étroite préconisée par Weber entre «le savant et le politique » est pleinement mise en œuvre dans ce type d'agenda où l'« engagement préalable » du chercheur est clair: dès l'introduction, Jacques Cherblanc, éditeur de l'ouvrage et directeur du Lerars, décrit la démarche du laboratoire comme "orientée vers la reritualisation des actions à l'égard du deuil, de la maladie et de la naissance ».

4 Parler de « reritualisation » induit l'idée d'une perte de rituel ; or c'est précisément le dynamisme du rituel que défendent les auteurs de ce volume. Le rituel ne serait pas mort, il se serait déplacé : individualisé, hybridisé, mais surtout, à en croire les thèmes abordés ici, sécularisé. Ainsi, les lieux décrits dans les différentes contributions comme des lieux potentiellement rituels : école, hôpital, entreprise ou morgue, tendent à indiquer que pour les auteurs de cet ouvrage, les liminalités contemporaines peuvent s'exercer dans des institutions laïcisées, diversifiées et spécialisées.

5 On peut regretter alors l'équivalence qui semble être faite entre le concept de « rites contemporains » qu'annonce le titre, et celui de "rites séculiers » qui représente le contenu de l'ouvrage : c'est passer sous silence les créativités rituelles contemporaines sur la scène religieuse, des mega-churches à la néo-Kabbale en passant par le soufisme occidental et les nouveaux mouvements religieux. Le projet a cependant le mérite de tenter d'étendre l'analyse rituelle au-delà de son champ religieux traditionnel et de chercher à explorer de nouveaux terrains et de nouvelles pratiques.

6 En se focalisant sur des variantes de rites de passage et de guérison comme l'intégration universitaire, l'union civile ou la thérapie, l'ouvrage prend ainsi le parti d'une définition fonctionnaliste extensive du rite s'inscrivant dans la lignée des travaux 
d'Albert Piette sur les Religiosités séculières, de Claude Rivière sur les Rites profanes, ou encore d'Ervin Goffmann sur Les rites d'interaction dans la vie quotidienne.

7 Ainsi, Jacques Cherblanc distingue deux acceptions principales des notions de rites et de symboles : les définitions traditionnelles descriptives, et celles, pour lui d'avantage à même de saisir la modernité séculière, les définitions explicatives de la fonction sociale de rites. Dans la même perspective, Denis Jeffrey, spécialiste québécois des ritualités contemporaines et scolaires, s'emploie à déconstruire ce qu'il identifie comme les préconceptions concernant les notions de "rites et ritualisations », en particulier leur rattachement "naïf» et trop fréquent aux notions de "religieux», "sacré », «éthique », etc. Par conséquent, sa proposition d'« élargir notre regard sur les rites » implique que "toutes les activités [puissent] être étudiées sous l'angle de leur ritualisation » (p. 54).

Après cette introduction «théorique " annonciatrice d'un parti-pris extensif de la notion de rite, le corps de l'ouvrage consiste en une partie pratique composée d'études de cas et subdivisée entre "rites, symboles et passages» et "rites, symboles et institutions ». Un tel découpage souligne la bi-dimensionnalité du rite comme à la fois individuel et institutionnel.

Dans un premier temps, les contributions se focalisent sur l'individu et sur l'efficacité subjective du rituel lors de différents moments redéfinis comme les nouveaux grands «passages » de la vie : les études, la thérapie, et la mort.

10 S'appuyant sur deux études de cas pré ou postscolaires : les « bals de finissants » et les " initiations universitaires ", David Harvengt explore «la relation particulière entre l'individualité et la collectivité » (p. 59). Il y souligne le paradoxe de ces coutumes qui, pour être optionnelles et subjectivement interprétées ad hoc, n'en sont pas moins selon lui, de véritables « rites de passage ». Moins convaincante est l'équivalence faite entre la notion de "film culte» et celle de ritualité adolescente (Brice Courty, Jocelyn Lachance et Philippe St-Germain, p. 73). Une seule contribution aborde la notion de " spiritualité » chez les étudiants. Sivane Hirsch observe sans surprise son caractère hybride, bricolé, sécularisé et individualiste. On reste cependant plus sceptique sur la requalification de certaines activités, comme la «culture» ou le "camping», en " spiritualités ", tout comme de l'interprétation du « récit » comme " rite » (p. 82).

11 La seconde thématique aborde la thérapie, un domaine d'activité qui s'est considérablement accru ces dernières décennies, et qui, dans l'optique de cet ouvrage, est considéré sous l'angle du rite de passage. À partir de l'étude de cas d'un patient auquel il a proposé le processus du "rêve éveillé ", Louis Charles-Lavoie entend décrire la psychothérapie comme "passage ritualisé ». S'appuyant sur la structure " rituelle » de la rencontre thérapeutique, sur les liens - controversés - opérés par Jung entre spiritualité et personnalité, et sur la résurgence de "symboles religieux » dans le discours du patient, l'auteur conclut, peut-être un peu rapidement, que " psychothérapie a pris le relais des rites de passage traditionnels » (p. 104).

12 Dans le même esprit, mais à rebours, c'est sous forme de "rite de passage » que Francine Gaudreault entend offrir une thérapie aux femmes victimes d'inceste. $L^{\prime}$ ' intervention rituelle et symbolique » (p.107) qu'elle propose consiste en nouveaux rites de guérison sur-mesure, mobilisant symboles à la fois religieux et personnels en vue de leur efficacité pratique sur les traumatismes individuels. L'importance du thème de la thérapie dans cette rubrique sur "les rites de passage à l'âge adulte » - au détriment de thèmes comme le mariage, la naissance ou même le divorce, met en 
lumière le rôle qu'a pris le « développement personnel » au sens large (de la méditation à la psychanalyse en passant par l'acupuncture et les groupes de rétablissement «12 étapes ») dans les sociétés occidentales contemporaines.

13 La seconde partie " pratique » entend examiner le rapport entre individu et Institution au travers de trois cas hétérogènes : une grande école, la mairie, et l'entreprise.

Ce sont les alternatives au mariage religieux qu'Antoine Mandret Degeilh passe en revue dans « S'unir à la mairie ». Là encore, l'institution séculière est présentée comme le garde-fou d'une baisse générale de la ritualité sociale, un lieu où la consécration publique et le faste rituel sont encore - même discrètement - recherchés. La mairie est ainsi décrite comme lieu transitoire symbolique, entre démarche "prospective » des couples gays en recherche d'officialisation, et démarche "alternative" des couples hétérosexuels désireux de plus d'informalité dans l'engagement.

La dernière catégorie de "rites de passage" concerne la mort. Focalisées respectivement sur les souhaits des souscripteurs de contrats obsèques (Bérangère Véron) et sur les pratiques des thanatologues (Jacques Cherblanc), les deux contributions présentées confirment les analyses contemporaines sur la modernité religieuse: comme toute autre dimension sociale, la mort se voit privatisée et sécularisée. Notamment, la subjectivisation des rites se traduit par une standardisation des pratiques: différenciée, chez les souscripteurs de contrats obsèques, selon un capital social proportionnel au niveau de créativité et d'autonomie par rapport à la tradition religieuse; et soumise à la loi de l'offre et de la demande dans le cas de la thanatologie, décrite comme une pratique substitutive à l'institution religieuse funéraire selon trois piliers : « symbolisme », « ritualisme », et « capitalisme » (p. 147).

En somme, il ressort de toutes ces études de cas un constat d'autonomisation de l'individu par rapport aux institutions. L'accent est mis sur l'expérience personnelle de l'étudiant, du patient en psychothérapie, de la personne âgée face à sa finitude. Par conséquent, le récipiendaire devient de plus en plus le concepteur de son propre rituel, tandis que l'institution religieuse devient, le cas échéant, une ressource mobilisable ad hoc en tant que réservoir de symboles. En ce sens, les travaux du sociologue américain Peter Berger sur l'« impératif hérétique » de l'individu contemporain (1979) ou de la sociologue française Danièle Hervieu-Léger sur la place du religieux comme ressource de tradition dans des sociétés sécularisées marquées par la multiplication de "petits récits croyants individuels" (2005), notamment sous forme de processus d'«autolégitimation» ou de "validation mutuelle du croire» (1999), auraient pu s'avérer heuristiques. De même, les Performance Studies, auraient gagné à être mobilisées, dans une étude qui se focalise sur la fonction performative du rite pour le qualifier comme tel. D'autant que la qualification en "rites » de certaines pratiques reste peu convaincante, notamment dans les cas de la thérapie, du récit et du cinéma. Elle implique une redéfinition des notions de « rite» et de "spiritualité », qui semble parfois manquer de distanciation quant à leur acception dans le langage commun : l'expression du « rituel du café du matin » par exemple, ne signifie pas que la recherche doive qualifier ces habitudes en rites. Seul l'article de Ghislaine Gallenga, isolé tant par son objet : l'entreprise, que par son angle, critique l'extension de la notion de rite à tous les domaines de la vie contemporaine. Enfin, d'autres domaines des ritualités contemporaines auraient gagné à être abordés, comme la question de la ritualité dans le sport ou dans le star-system. 
17 L'ouvrage a cependant le mérite d'ouvrir de nouvelles perspectives et de faire ressortir les tendances actuelles en matière de demande de ritualisation. Ainsi, la place marginale réservée aux rites d'union, de naissance, et aux rites religieux, tend à faire ressortir des nouvelles zones de priorité rituelle dans les sociétés occidentales contemporaines : l'organisation de sa vie professionnelle, personnelle, et de sa fin. Les réflexions sur le mourir occidental contemporain demeurent sans aucun doute, par la rigueur de leur traitement et la finesse de leur analyse, les contributions essentielles de cet ouvrage. Celui-ci offre donc, comme c'était son objet, une introduction utile et accessible à la question du rite et de ses applications dans les sociétés occidentales contemporaines. 